\title{
Carrier-induced refractive index change and optical absorption in wurtzite InN and GaN: Full- band approach
}

\author{
Ceyhun Bulutay, ${ }^{1}{ }^{*}$ Cem Murat Turgut, ${ }^{1}$ and N. A. Zakhleniuk ${ }^{2}$ \\ ${ }^{1}$ Department of Physics, Bilkent University, Ankara 06800, Turkey \\ ${ }^{2}$ School of Computer Science and Electronic Engineering, University of Essex, Wivenhoe Park, Colchester CO4 3SQ, United Kingdom
}

(Received 12 November 2009; revised manuscript received 22 February 2010; published 16 April 2010)

\begin{abstract}
Based on the full band electronic structure calculations, first we consider the effect of $n$-type doping on the optical absorption and the refractive index in wurtzite $\mathrm{InN}$ and $\mathrm{GaN}$. We identify quite different dielectric response in either case; while $\mathrm{InN}$ shows a significant shift in the absorption edge due to $n$-type doping, this is masked for GaN due to efficient cancellation of the Burstein-Moss effect by the band gap renormalization. Moreover, for high doping levels the intraband absorption becomes significant in InN. For energies below $1 \mathrm{eV}$, the corresponding shifts in the real parts of the dielectric function for $\mathrm{InN}$ and $\mathrm{GaN}$ are in opposite directions. Furthermore, we observe that the free-carrier plasma contribution to refractive index change becomes more important than both band filling and the band gap renormalization for electron densities above $10^{19} \mathrm{~cm}^{-3}$ in $\mathrm{GaN}$, and $10^{20} \mathrm{~cm}^{-3}$ in InN. As a result of the two different characteristics mentioned above, the overall change in the refractive index due to $n$-type doping is much higher in $\mathrm{InN}$ compared to GaN, which in the former exceeds $4 \%$ for a doping of $10^{19} \mathrm{~cm}^{-3}$ at $1.55 \mu \mathrm{m}$ wavelength. Finally, we consider intrinsic $\mathrm{InN}$ under strong photoexcitation which introduces equal density of electron and holes thermalized to their respective band edges. The change in the refractive index at $1.55 \mu \mathrm{m}$ is observed to be similar to the $n$-doped case up to a carrier density of $10^{20} \mathrm{~cm}^{-3}$. However, in the photoexcited case this is now accompanied by a strong absorption in this wavelength region due to $\Gamma_{5}^{v} \rightarrow \Gamma_{6}^{v}$ intravalence band transition. Our findings suggest that the alloy composition of $\operatorname{In}_{x} \mathrm{Ga}_{1-x} \mathrm{~N}$ can be optimized in the indium-rich region so as to benefit from high carrierinduced refractive index change while operating in the transparency region to minimize the losses. These can have direct implications for InN-containing optical phase modulators and lasers.
\end{abstract}

DOI: 10.1103/PhysRevB.81.155206

PACS number(s): 78.20.Ci, 78.20.Bh, 78.40.Fy

\section{INTRODUCTION}

The research efforts on $\mathrm{InN}$ and In-rich InGaN have been intensifying worldwide. As well as their traditional applications on solid-state lightning and lasers, new possibilities begin to flourish such as photovoltaics and chemical sensing. ${ }^{1}$ From the electronic structure point of view, InN has a number of unique properties. ${ }^{2}$ Its band gap for the wurtzite phase is commensurate with the $1.55 \mu \mathrm{m}$ wavelength for fiber optics when it forms an alloy with a small amount of GaN. Furthermore, the small conduction band effective mass enables interesting band-filling effects. Even though the latter giving rise to Burstein-Moss effect ${ }^{3,4}$ was the center of focus in the recent re-assessment of the high quality $\mathrm{InN}$ samples, ${ }^{5-8}$ its device implications have not been given the full attention it deserved. Among the viable applications are the carrier-induced optical phase modulators, ${ }^{9}$ and tunable Bragg reflector and filters. ${ }^{10,11}$ These devices are expected to play essential role, for instance, for the signal processing directly in the optical domain in the future high bit rate optical communication networks.

It was known from the early days of edge-emitting semiconductor lasers that carrier-induced refractive change affects the optical beam quality, ${ }^{12}$ such as mode guiding along the junction plane, self-focusing, filament formation, frequency chirping under direct modulation. ${ }^{13,14}$ The refractive index tunability by carrier injection was studied in the previous decade for the Indium-Group-V semiconductor compounds and alloys other than InN. ${ }^{15-17}$ In the case of InGaN laser structures, there have been recent theoretical ${ }^{18}$ and experimental ${ }^{19,20}$ studies on the carrier-induced refractive index change and the linewidth enhancement factor. However, these studies considered only Ga-rich limit of InGaN alloys.

The purpose of this work is to offer a comparative theoretical account of carrier-induced refractive index change in both $\mathrm{InN}$ and $\mathrm{GaN}$, so as to form a basis for the In-rich InGaN alloys. Primary attention is given to $n$-type doping which is the prevalent type among the routinely grown samples. ${ }^{1}$ For the case of $\mathrm{InN}$, the photoexcited bipolar carrier-induced dielectric effects are also examined. The crux of our study is based on the Bennett-Soref-Del Alamo approach $^{15}$ which includes band-filling, band gap renormalization (BGR) and plasma contributions. However, rather than a model band structure, we employ a rigorous full band technique to represent the band filling; preliminary account of this work was given in Ref. 21. In Sec. II we present the theoretical details, this is followed by our results in Sec. III. A self-critique of our model and further discussions are provided in Sec. IV, ending with our conclusions in Sec. V. The Appendix contains our empirical pseudopotential parameters used for wurtzite InN.

\section{THEORY}

First, we would like to describe the electronic structure on which our computations are based. In the case of GaN, we use the local empirical pseudopotential method that we fitted to the existing experimental data, putting special emphasis on the conduction band behavior. ${ }^{22}$ For InN, we use the nonlocal empirical pseudopotential method, treating the angular- 


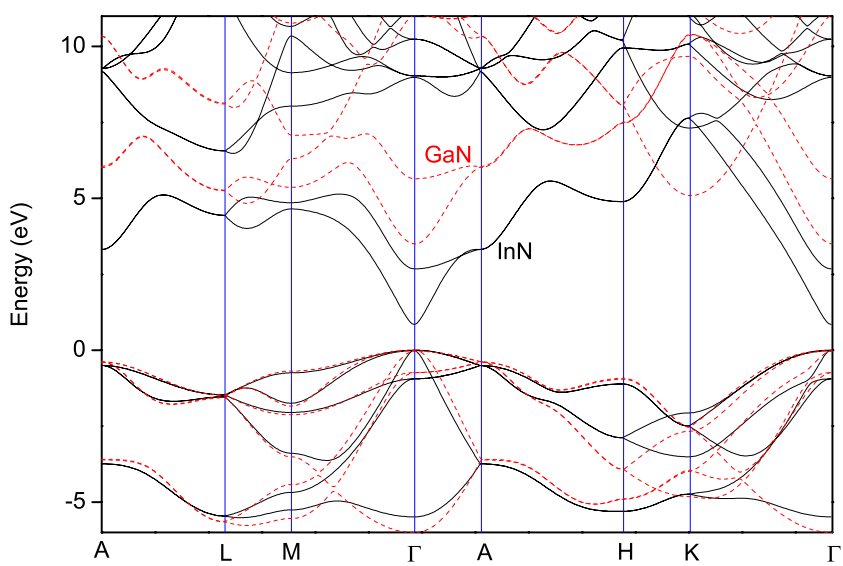

FIG. 1. (Color online) The empirical pseudopotential band structures for $\mathrm{InN}$ (solid) and $\mathrm{GaN}$ (dashed) used in this work.

momentum channels $p$ and $d$ for $\mathrm{In}$, and $p$ for $\mathrm{N}$ as nonlocal. ${ }^{23}$ Further details are given in the Appendix section. The corresponding band gap for InN comes out as $0.85 \mathrm{eV}$ which agrees very well with the recent first-principles results ${ }^{24,25}$ but it is higher than the $0.64 \mathrm{eV}$ value established experimentally. ${ }^{1}$ Figure 1 shows the band structures of wurtzite InN and GaN.

As the Burstein-Moss effect ${ }^{3,4}$ primarily shifts the absorption edge due to band filling, the ultimate quantity to be computed is the imaginary part of the optical (i.e., the long wavelength limit) dielectric tensor which is given in Gaussian units by ${ }^{26}$

$$
\begin{aligned}
\operatorname{Im}\left\{\epsilon^{a b}(\omega)\right\}= & \frac{e^{2}}{\pi} \sum_{v, c} \int_{\mathrm{BZ}} d \mathbf{k} r_{v c}^{a}(\mathbf{k}) r_{c v}^{b}(\mathbf{k}) \delta\left(E_{c}(\mathbf{k})-E_{v}(\mathbf{k})\right. \\
& -\hbar \omega),
\end{aligned}
$$

where $a, b$ are the Cartesian indices. $r_{v c}^{a}(\mathbf{k})$ $=p_{v c}^{a}(\mathbf{k}) /\left[i m_{0} \omega_{v c}(\mathbf{k})\right]$, where $p_{v c}^{a}(\mathbf{k})$ is the momentum matrix element, $m_{0}$ is the free electron mass, $\omega_{v c}(\mathbf{k}) \equiv \omega_{v}(\mathbf{k})$ $-\omega_{c}(\mathbf{k})$, where $\hbar \omega_{n}(\mathbf{k}) \equiv E_{n}(\mathbf{k})$ is the energy of the band $n$, at the wave vector $\mathbf{k}$. The specific band label $v(c)$ represents filled (unfilled) bands, where for the clean cold semiconductors, the band occupation factors are taken as either one or zero. The volume integration in Eq. (1) is over the first Brillouin zone (BZ) which can be reduced to the irreducible BZ using the symmetry relations of the BZ (see, Fig. 2).

The Dirac delta term in Eq. (1) automatically reduces the volume integration to a surface formed by the $\mathbf{k}$ points which allow direct (vertical) transitions from a filled state to an unfilled state with an energy difference corresponding to the chosen photon energy. Such surface integrations routinely appear in the density of states, effective mass and response function calculations. ${ }^{27}$ They can be efficiently calculated numerically using the Lehmann-Taut method. ${ }^{28}$ This technique has also been shown to work for the second-order nonlinear optical response function ${ }^{29}$ and also for the full band phonon scattering rate calculation. ${ }^{22}$ In this work, for the required accuracy, we divide the irreducible BZ into a mesh of $40 \times 40 \times 40$ along the basal plane and the $c$-axis directions (see, Fig. 2). In Fig. 3, we show the imaginary part of
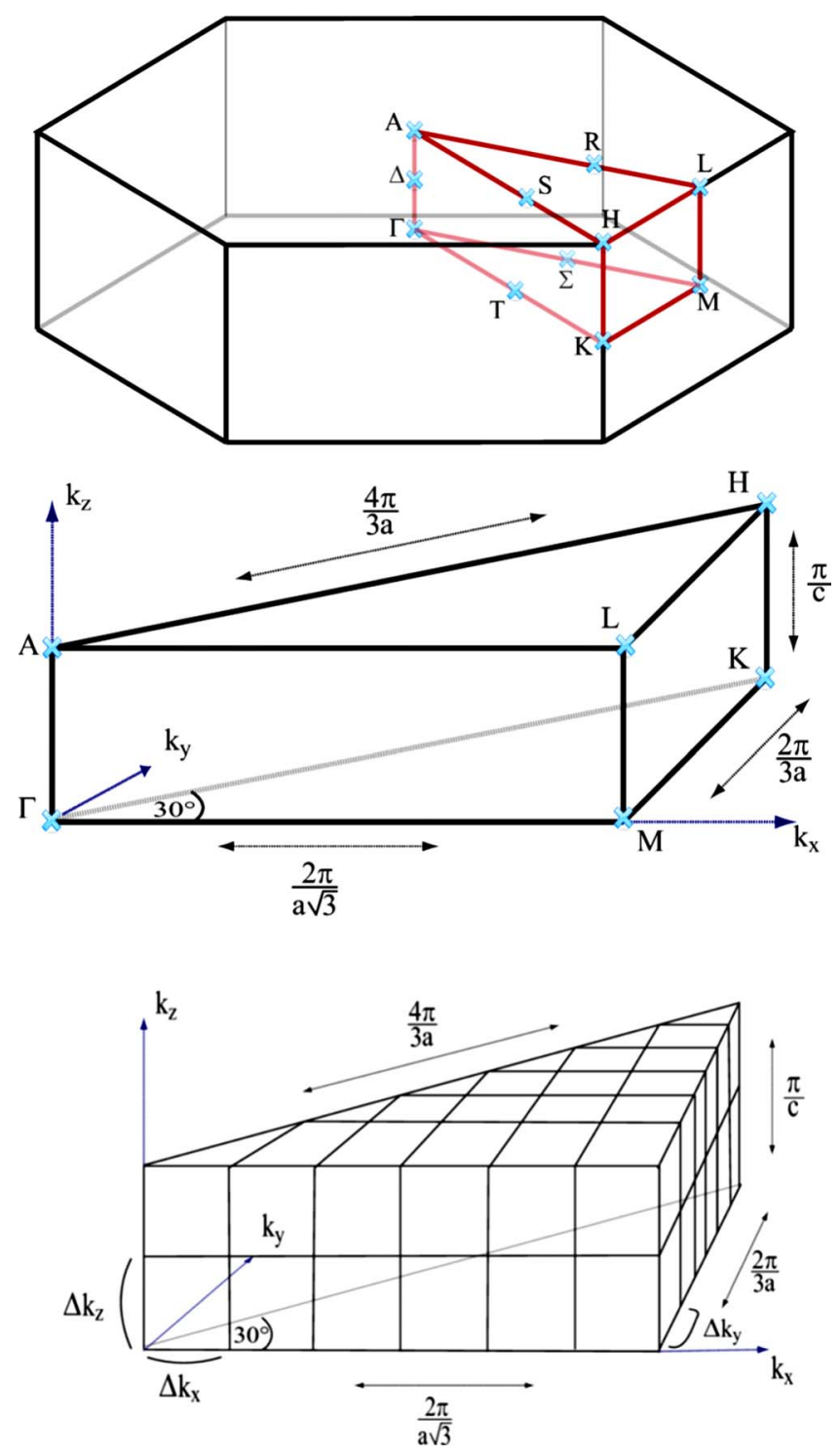

FIG. 2. (Color online) Schematic (not to scale) of the Brillouin zone of the wurtzite structure (top), its irreducible Brillouin zone (middle), and its tessellation (bottom); each volume is further divided into tetrahedra (not shown). $a$ and $c$ are the lattice constants in the basal plane and perpendicular directions, respectively.

the optical dielectric function for the intrinsic (i.e., undoped) InN and GaN. The ordinary tensor component which corresponds to the electric field parallel to basal plane of the hexagonal crystal (i.e., in-plane) polarization is shown. For GaN, we observe a discrepancy between the theoretical and experimental $^{31}$ values close to the band edge. On the other hand, our theoretical result for $\mathrm{InN}$ matches well with the rigorous first-principles studies, ${ }^{32,33}$ and also in reasonable agreement with the experiment. ${ }^{30}$

Another physical mechanism that accompanies bandfilling is the BGR due to many-body interactions among the carriers. ${ }^{34}$ In highly $n$-doped $\mathrm{InN}$ samples it is observed that this partially cancels the Burstein-Moss effect. ${ }^{7}$ The electronelectron and electron-ion contributions within the random phase approximation are given in Gaussian units by ${ }^{7,34}$ 


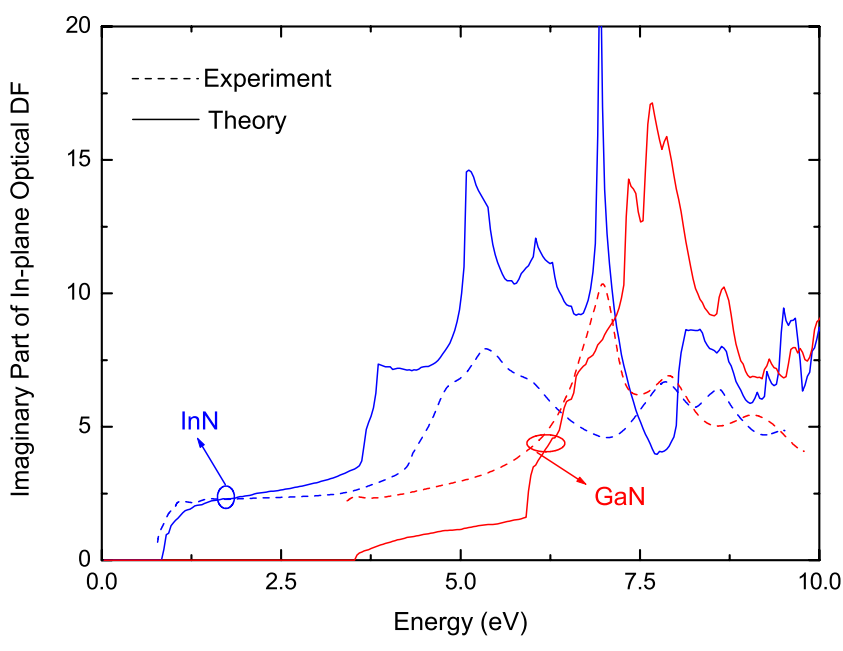

FIG. 3. (Color online) Imaginary part of the in-plane optical dielectric function for the intrinsic $\mathrm{InN}$ and $\mathrm{GaN}$, comparing our theoretical (solid) results with the experimental (dashed) data of Refs. 30 and 31.

$$
\begin{gathered}
\Delta E_{e-e}=-\frac{2 e^{2} k_{F}}{\pi \epsilon_{s}}-\frac{e^{2} k_{T F}}{2 \epsilon_{s}}\left[1-\frac{4}{\pi} \arctan \left(\frac{k_{F}}{k_{T F}}\right)\right], \\
\Delta E_{e-i}=-\frac{4 \pi e^{2} N}{\epsilon_{s} a_{B}^{*} k_{T F}^{3}},
\end{gathered}
$$

where $N$ is the electron (ion) density and $\epsilon_{s}$ is the static permittivity for which we use the experimental value of 9.5 (15.3) for $\mathrm{GaN}(\mathrm{InN}){ }^{35,36} \quad k_{F}=\left(3 \pi^{2} N\right)^{1 / 3}$ and $k_{T F}$ $=2 \sqrt{k_{F} /\left(\pi a_{B}^{*}\right)}$ are, respectively, the Fermi and Thomas-Fermi wave numbers, $a_{B}^{*}=0.529 \epsilon_{s} m_{0} / m^{*}$ is the effective Bohr radius in $\AA, m^{*}$ is the conduction band effective mass. The latter quantity rapidly deviates with increase of energy from its band edge value, especially in the case of InN. Therefore, in these expressions we use an energy-dependent density of states effective mass value corresponding to the level of band-filling. Figure 4 shows for the conduction band these values used in this work for InN and GaN. The curve for InN is taken from the first-principles results. ${ }^{24}$ For GaN, it has been computed using the same Lehmann-Taut method, but with a finer grid than used above, such as $60 \times 60 \times 60$ or denser. In the case of photoexcitation which we consider for InN, actually we have a two-component quantum system (i.e., electron and hole gases). In the interests of simplicity, we adapt Eq. (2) by replacing the electron effective mass with the reduced electron-hole effective mass, $\mu_{e h}$ given by $\mu_{e h}^{-1}=m_{e}^{-1}+m_{h}^{-1}$ where $m_{e}$ and $m_{h}$ are the energy-dependent electron and hole effective masses, respectively. The latter is almost constant for the densities dealt in this work and therefore is taken to be $0.84 m_{0}$ for $\mathrm{InN}$.

Having determined the imaginary dielectric function of a semiconductor with partially filled band(s), subject to BGR, the next aim is to compute their effect on the refractive index. Their interrelation is established by the causality principle which has to be satisfied by any physical response function. ${ }^{37}$ In mathematical terms, this is governed by the Kramers-Kronig relation; for the real part, the corresponding

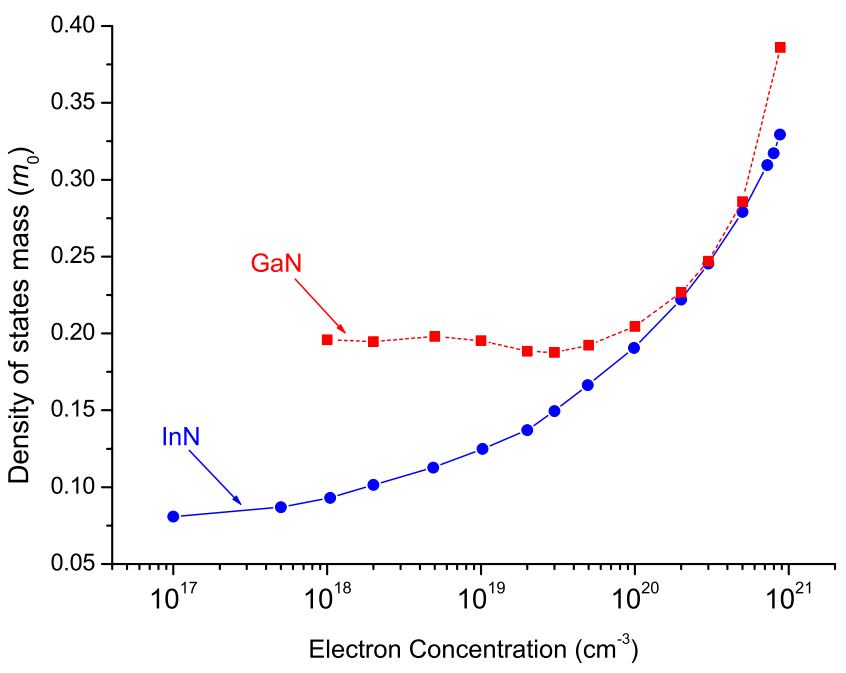

FIG. 4. (Color online) The density-of-states effective mass variation as a function of band-filling for the conduction band electrons in InN and GaN. The latter is computed in this work, whereas the former is taken from Ref. 24.

expression, suppressing the Cartesian indices is given by

$$
\operatorname{Re}\{\epsilon(\omega)\}=1+\frac{2}{\pi} \mathcal{P} \int_{0}^{\infty} \frac{\omega^{\prime} \operatorname{Im}\left\{\epsilon\left(\omega^{\prime}\right)\right\}}{\omega^{\prime 2}-\omega^{2}} d \omega^{\prime} .
$$

Computationally, it requires the knowledge of the imaginary part of the dielectric function for all energies; we have verified the convergence of our results by including 60 bands and accounting for the higher energy behavior (above $30 \mathrm{eV}$ ) analytically with a $1 / \omega^{2}$ fall off.

Based on the mechanisms described above, carrier injection essentially leads to a change in the refractive index. Another significant contribution to the refractive index change is caused by the plasma absorption of the free carriers: electrons in the conduction band for the $n$-doped case or the electrons and holes for the photoexcited case. Its resultant contribution can be expressed in convenient units as ${ }^{15}$

$$
\Delta n_{\text {plasma }}=-\frac{6.9 \times 10^{-22}}{n_{0} m_{r}^{*}} \frac{N\left[\mathrm{~cm}^{-3}\right]}{E^{2}[\mathrm{eV}]},
$$

where $n_{0}$ is the refractive index of the intrinsic semiconductor, $E[\mathrm{eV}]$ is the energy in $\mathrm{eV}, N\left[\mathrm{~cm}^{-3}\right]$ is the free-carrier density in $\mathrm{cm}^{-3}$ and $m_{r}^{*}$ is the effective mass in units of $m_{0}$; in the photoexcited case, for the latter two we use the electronhole density and the effective mass $\left(\mu_{e h}\right)$, respectively.

\section{RESULTS}

\section{A. $n$-doped case in InN and GaN}

We first start with the $n$-doped case which is quite easily achieved (sometimes even unintentionally) in InN and GaN and, we explore the filling of the conduction band by electrons fully ionized from their dopants. The electron concentrations up to about $7 \times 10^{20} \mathrm{~cm}^{-3}\left(9 \times 10^{20} \mathrm{~cm}^{-3}\right)$ are considered marking the threshold beyond which the next higher conduction band starts to be filled for $\mathrm{InN}(\mathrm{GaN})$. Figure 5 


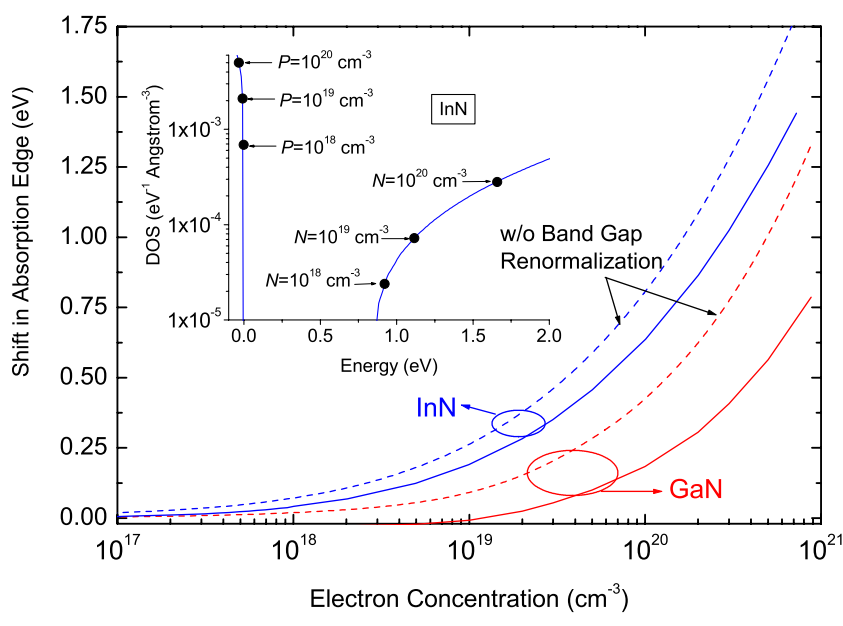

FIG. 5. (Color online) The shift in the absorption edge by $n$-type doping for $\mathrm{GaN}$ and $\mathrm{InN}$ due to conduction band filling without (dashed) and with (solid) BGR accounted. The inset shows the density of states (DOS) for InN together with the band-filling levels on the valence and conduction bands in the absence of BGR.

shows the shift in the absorption edge due to Pauli blocking of the filled portions of the lowest conduction band. A marked contrast revealed with this plot is that the absorption edge shift in $\mathrm{InN}$ is much more significant compared to GaN. The disparity between the two materials' conduction band edge density of state behavior plays a role here. While in InN the band-filling effect is quite strong, in the case of GaN this becomes smaller which is moreover effectively canceled by the BGR.

Yoshikawa et al. have used temperature-dependent photoluminescence spectroscopy to separate BGR and band-filling effects in Si doped GaN. ${ }^{38}$ Figure 6 illustrates the comparison of our $n$-doped $\mathrm{GaN}$ results with this work for the reduced band gap, i.e., due to BGR, as well as for the optical gap which corresponds to the absorption edge including the

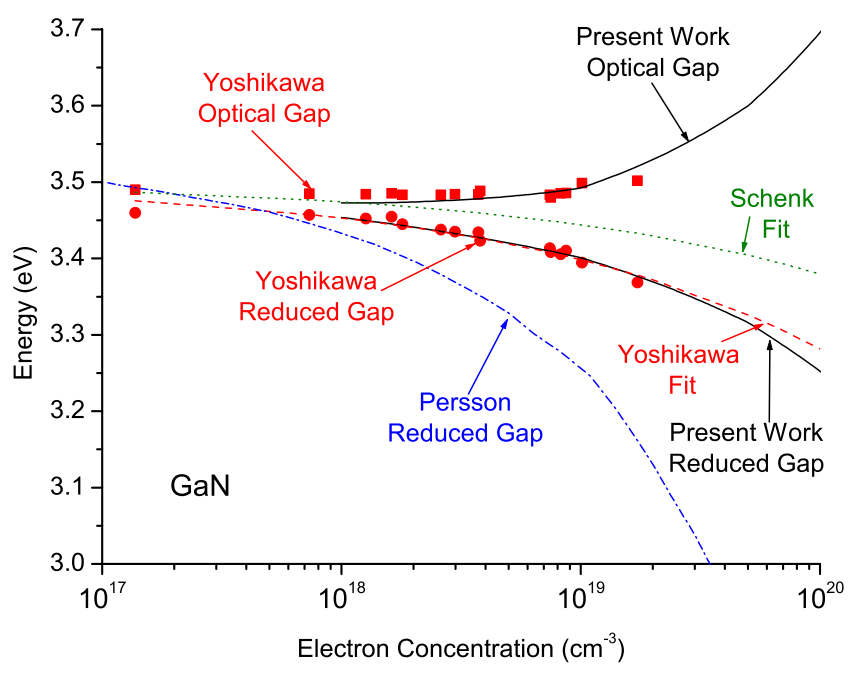

FIG. 6. (Color online) The comparison for the $n$-doped GaN, the reduced gap and the optical gap values (see text) with Yoshikawa et al. (Ref. 38), Schenk et al. (Ref. 39), and Persson et al. (Ref. 40). The band gap of intrinsic GaN is taken as $3.5 \mathrm{eV}$.

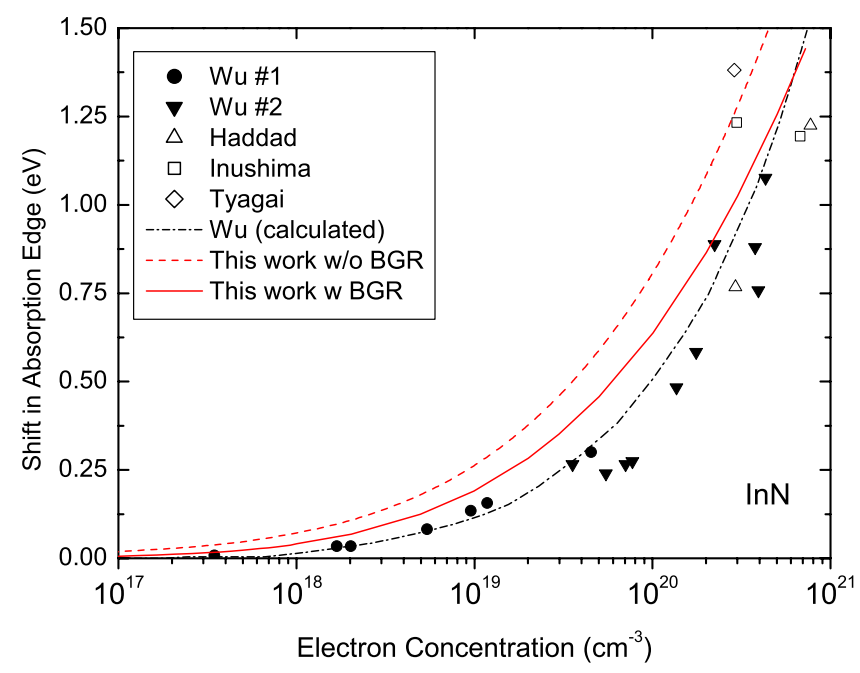

FIG. 7. (Color online) The comparison of measured and calculated shifts in the absorption edge in $\mathrm{InN}$ with the calculation and measurements from Wu (Ref. 8), together with other data mentioned in that work: Haddad (Ref. 43), Inushima (Ref. 42), and Tyagai (Ref. 41).

BGR and Burstein-Moss effects. The dashed line is the fitted expression, $-4.72 \times 10^{-8} n^{1 / 3} \mathrm{eV}$ offered by Yoshikawa et al. for the former. Very recently Schenk et al. relying on band edge luminescence peak and the line shape information have extracted an expression given by $-2.6 \times 10^{-8} n^{1 / 3} \mathrm{eV}$ for the BGR in $n$-doped GaN. ${ }^{39}$ While our result closely agrees with the Yoshikawa data, Schenk's expression is in disagreement with these. In Fig. 6, also a much more elaborate theoretical analysis for the BGR is shown which however deviates from the rest of these results. ${ }^{40}$ We believe that further studies are required to reach to a consensus over the BGR expression for $\mathrm{GaN}$. The situation is more harmonious for $\mathrm{InN}$. The comparison of our computed absorption shift for InN with the measurements and calculation from Wu et al. ${ }^{8}$ together with other data mentioned in that work ${ }^{41-43}$ is provided in Fig. 7; it shows the overall agreement of our results with the literature.

The conduction band filling and the associated BGR directly affect the imaginary part of the dielectric functions through the shift in the absorption edge deeper into the conduction band. This is illustrated for the ordinary components (i.e., the electric field lying on the crystal basal plane) for different electron concentrations in Figs. 8 and 9 for $\mathrm{InN}$ and $\mathrm{GaN}$, respectively. It can be noticed for both cases, especially for larger dopings, that there is a nonzero absorption below the absorption edge, even down to zero energy. This is due to intraband absorption within the conduction band (see the upper inset in Fig. 8). The same behavior also appears in the measured InN samples. ${ }^{8}$

The real parts of the dielectric functions are obtained from the imaginary parts using the Kramers-Kronig relation in Eq. (4). The results are shown in Figs. 10 and 11 for $\mathrm{InN}$ and $\mathrm{GaN}$, respectively. First of all, the real part of dielectric function of InN is more sensitive to $n$-type doping. Furthermore, for low energies, the shifts are in opposite directions for InN and $\mathrm{GaN}$, so that as the electron concentration increases, the permittivity decreases for $\mathrm{InN}$, whereas it increases for $\mathrm{GaN}$. 


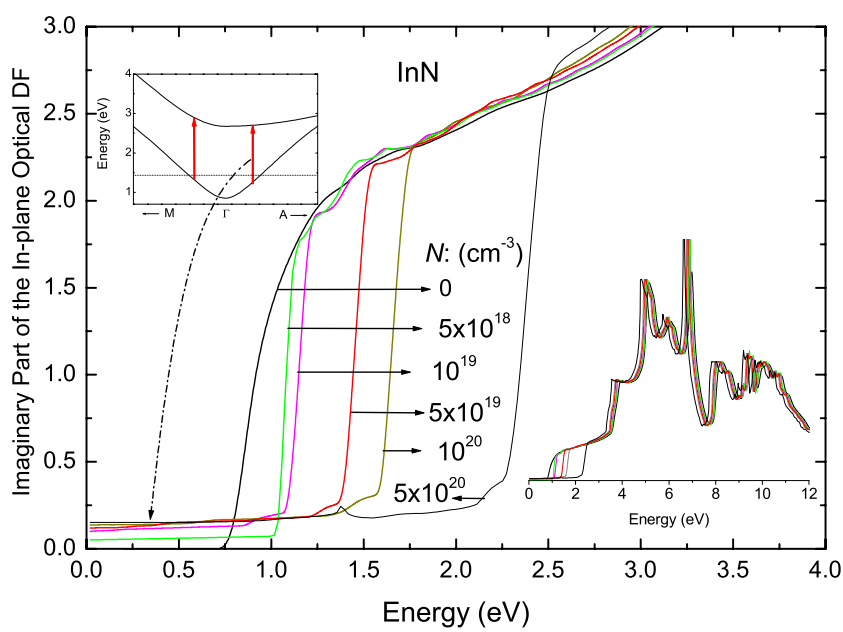

FIG. 8. (Color online) The calculated imaginary part of the inplane dielectric function (DF) of $\mathrm{InN}$ for different $n$-type doping densities. Lower inset shows the same graph for a wider energy scale. Upper inset indicates the responsible intraconduction band transitions for the low energy part of the spectra.

Note that these are only the electronic contributions to polarization as Eq. (1) does not include the ionic degrees of freedom. Nevertheless the ions do not directly play a role in the change in the refractive index which is focus of this work. As mentioned in the Theory section, one should also add the free-carrier plasma contribution to the refractive index [see, Eq. (5)]. This is separately shown in Fig. 12 which always has a negative contribution, and $\left|\Delta n_{\text {plasma }}\right|$ decreases as $1 / E^{2}$ with energy $E$.

The overall change in the refractive index, including all of the effects mentioned so far is shown in Fig. 13. Because of their technological importance, it is evaluated at the two fiber-optic communication wavelengths of 1.55 and $1.3 \mu \mathrm{m}$ which correspond to energies of 0.8 and $0.954 \mathrm{eV}$, respectively; the former is marked with dashed vertical arrows in Figs. 10 and 11. It can be observed that the refractive index change due to $n$-type doping is much higher in InN com-

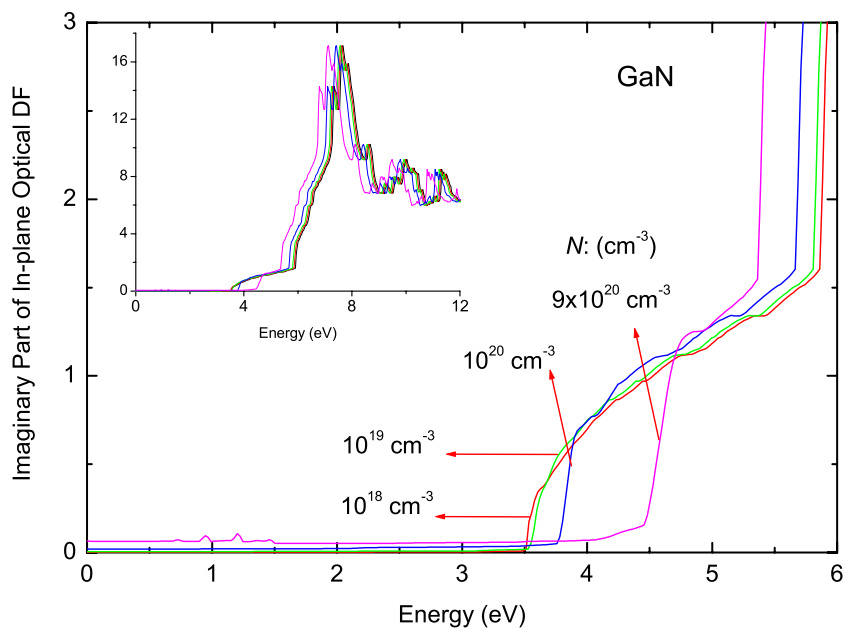

FIG. 9. (Color online) The calculated imaginary part of the inplane dielectric function of GaN for different $n$-type doping densities. Inset shows the same graph for a wider energy scale.

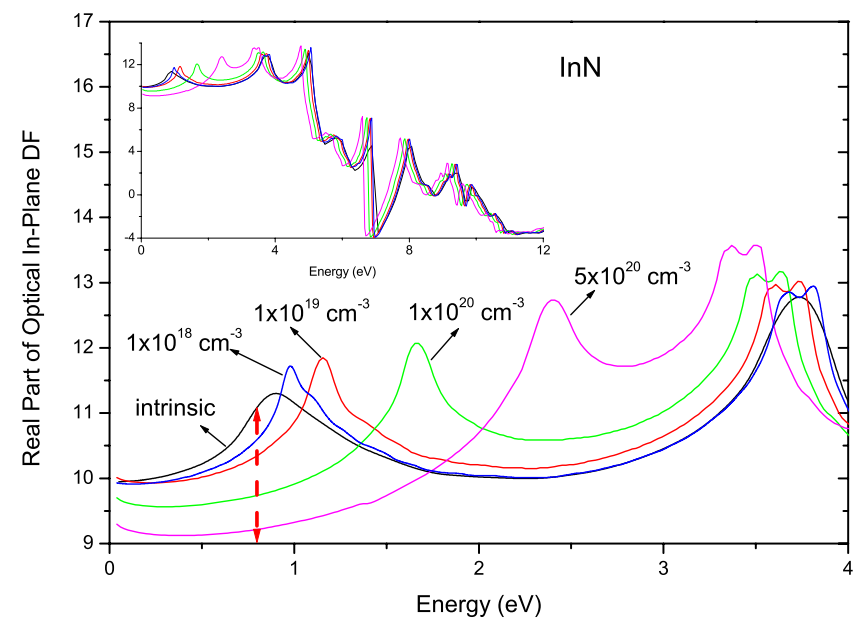

FIG. 10. (Color online) The calculated real part of the in-plane electronic dielectric function of $\mathrm{InN}$ for different $n$-type doping densities. The vertical red arrow marks the $0.8 \mathrm{eV}(1.55 \mu \mathrm{m})$ value. Inset shows the same graph for a wider energy scale.

pared to GaN for both wavelengths. There are several reasons behind this outcome. First of all there is a cancellation that occurs for GaN; as shown in Fig. 5, BGR very effectively cancels the Burstein-Moss shift up to a density of $10^{19} \mathrm{~cm}^{-3}$. The lack of such a cancellation in $\mathrm{InN}$ is because of the very strong band-filling effect as a result of small density of states of $\mathrm{InN}$ close to conduction band edge, see the inset in Fig. 5. Furthermore, contrary to the case in InN, the refractive index change in GaN due to band filling is positive up to the ultraviolet. This is canceled by the negative plasma contribution [cf., Eq. (5)], which actually dominates beyond a density of $10^{19} \mathrm{~cm}^{-3}$ in $\mathrm{GaN}$ and $10^{20} \mathrm{~cm}^{-3}$ in InN.

Note that at the $1.3 \mu \mathrm{m}$ wavelength the intrinsic as well as doped InN display substantial interband absorption (see, Fig. 8), hence, in Fig. 13 we used the following generaliza-

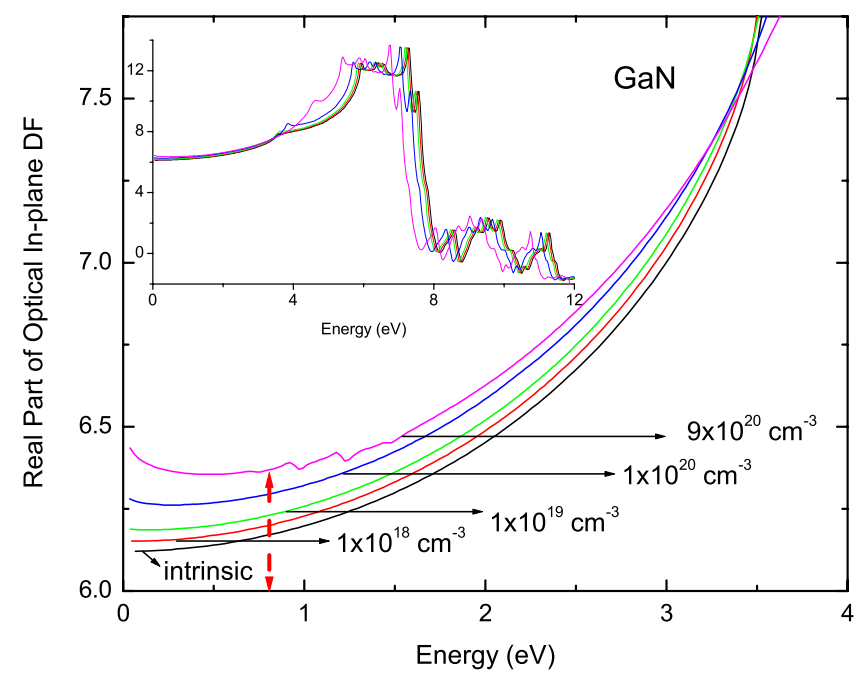

FIG. 11. (Color online) The calculated real part of the in-plane electronic dielectric function of $\mathrm{GaN}$ for different $n$-type doping densities. The vertical red arrow marks the $0.8 \mathrm{eV}(1.55 \mu \mathrm{m})$ value. Inset shows the same graph for a wider energy scale. 


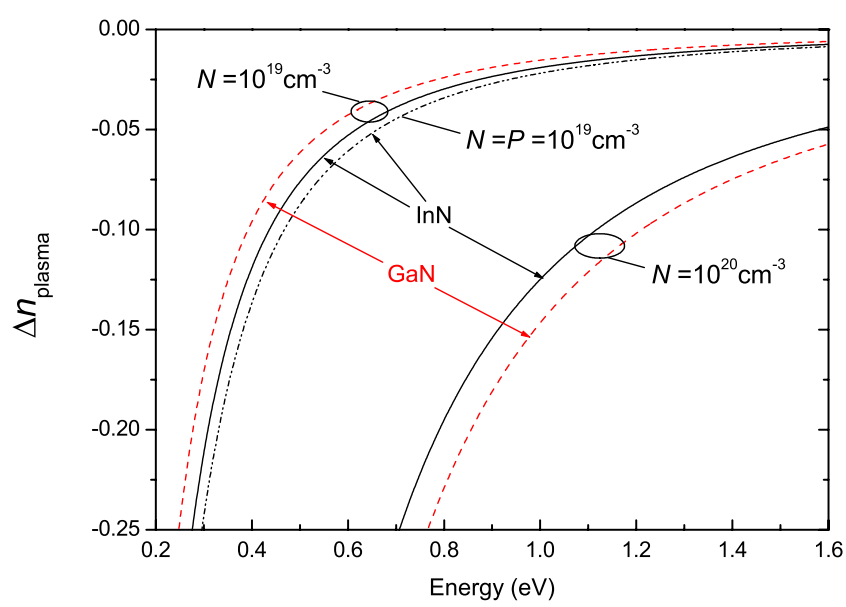

FIG. 12. (Color online) The comparison of the free-carrierinduced refractive index change, i.e., plasma contribution, [see, Eq. (5)] for two different $n$-type doping densities for $\mathrm{InN}$ and GaN. The photoexcited $N=P=10^{19} \mathrm{~cm}^{-3}$ bipolar case in $\mathrm{InN}$ is also included.

tion of the refractive index in the presence of loss: ${ }^{37}$

$$
n=\sqrt{\frac{\sqrt{\operatorname{Re}\{\epsilon\}^{2}+\operatorname{Im}\{\epsilon\}^{2}}+\operatorname{Re}\{\epsilon\}}{2} .}
$$

Because of this loss, $1.3 \mu \mathrm{m}$ case shows an overall reduction in the refractive index change compared to $1.55 \mu \mathrm{m}$ wavelength. As another curious point, the $n$-doped InN curve in the bottom panel of Fig. 13 starts from a positive value which requires an explanation. Due to the absorption in the $1.3 \mu \mathrm{m}$ wavelength, the refractive index also peaks around the $0.95 \mathrm{eV}$ range, particularly for the $10^{18} \mathrm{~cm}^{-3}$ density (cf. Fig. 10). Hence, the $n$-doped InN curve in Fig. 13 becomes positive for this density as its refractive index exceeds that of

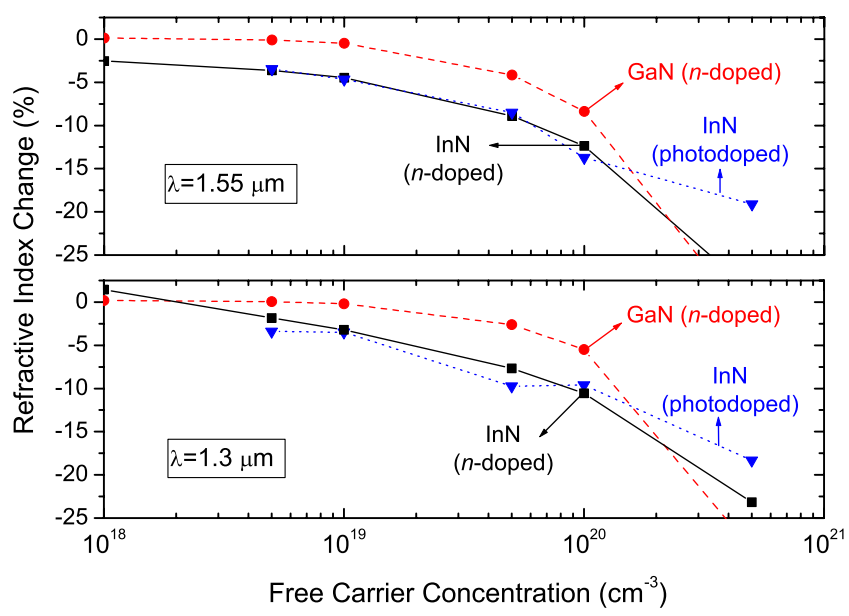

FIG. 13. (Color online) The percentage refractive index change with respect to intrinsic (i.e., no free carrier) case for $\mathrm{InN}$ and $\mathrm{GaN}$. All effects considered in this work are included, i.e., band-filling, BGR, and plasma contributions. Top panel shows the $1.55 \mu \mathrm{m}$ wavelength $(0.8 \mathrm{eV})$ and the bottom panel corresponds to the $1.3 \mu \mathrm{m}$ wavelength $(0.954 \mathrm{eV})$. See text, for the explanation of why $n$-doped $\mathrm{InN}$ curve in the bottom panel starts from a positive value. The lines are to guide the eyes.

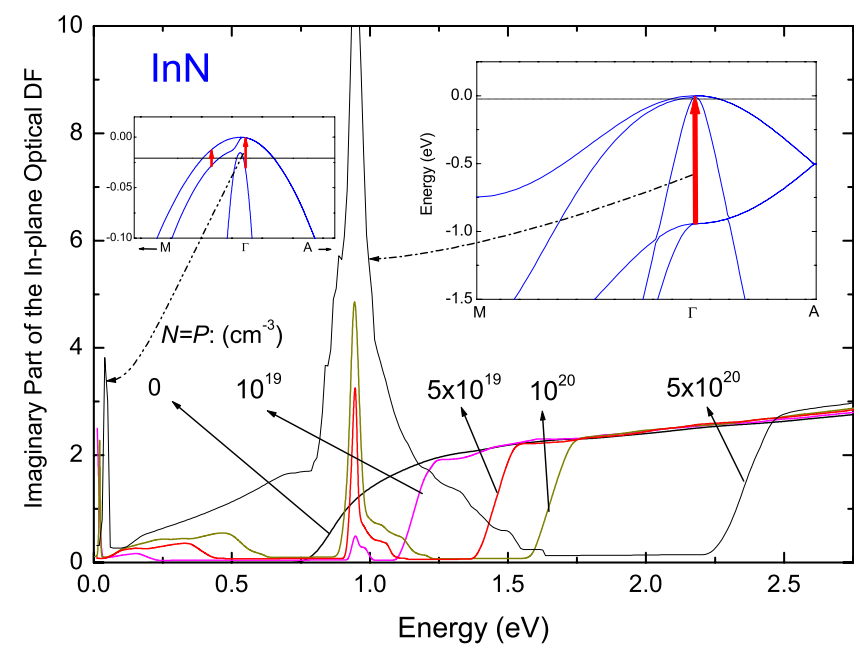

FIG. 14. (Color online) The calculated imaginary part of the in-plane dielectric function of $\mathrm{InN}$ for different electron-hole populations forming degenerate quasiequilibrium distributions. Insets show the intravalence band transitions that give rise to the new absorption channels around $0 \mathrm{eV}$ (left) and $0.94 \mathrm{eV}$ (right).

the intrinsic sample. Even though the band-filling and plasma contributions try to reduce it, they are not as effective at this density.

\section{B. Photoexcited case in InN}

We would like to compare the pronounced refractive index change in $n$-doped $\mathrm{InN}$ with the case where both electrons and holes are present. Since introducing holes with $p$-type doping is still a major technical challenge in $\mathrm{InN},{ }^{1}$ we consider photodoping ${ }^{44}$ where equal density of electrons and holes are generated by a strong photoexcitation. We further assume that these photocarriers quickly thermalize to their respective band edges and attain a degenerate quasiequilibrium distribution. Since the electron-hole pairs will eventually recombine within a time scale of $300-400 \mathrm{ps},{ }^{45}$ the quasicontinuous optical excitation should replenish this in order to sustain the assumed steady-state carrier distribution. The associated quasi-Fermi levels for different electron-hole densities are marked in the inset of Fig. 5 which clearly indicates that the contribution of the holes to the Burstein-Moss effect is negligible. In Fig. 14 we show the imaginary dielectric function at several electron-hole densities. Compared to the $n$-doped case in Fig. 8, we observe two new features around 0 and $0.94 \mathrm{eV}$ which are caused by the intravalence band transitions: the former is due to very low energy transitions at the valence band maximum (left inset in Fig. 14), whereas the latter is the result of the $\Gamma_{5}^{v} \rightarrow \Gamma_{6}^{v}$ transition (right inset in Fig. 14). It is the latter that makes a significant impact around $1.55 \mu \mathrm{m}(0.8 \mathrm{eV})$ and even more so at the $1.3 \mu \mathrm{m}$ wavelength $(0.954 \mathrm{eV})$. Therefore, we would like to assert that our band structure agrees quite well with the other firstprinciple studies ${ }^{25,32}$ which report the value $0.9 \mathrm{eV}$ with the quasiparticle correction for the $\Gamma_{5}^{v} \rightarrow \Gamma_{6}^{v}$ transition. As observed from Fig. 14, these new intravalence band channels refill the part of the spectra cleared by the Pauli blocking, undoing the Burstein-Moss effect. Also we can observe from 
Fig. 13 that below a carrier density of $10^{20} \mathrm{~cm}^{-3}$, the change in the refractive index at $1.55 \mu \mathrm{m}$ is seen to be very close to the $n$-doped case. However, it should be recalled that this is now accompanied by a strong absorption (cf., Fig. 14) which can hamper its possible device applications.

\section{SELF-CRITIQUE OF THE THEORETICAL MODEL AND DISCUSSIONS}

In this section we would like to discuss several aspects of the employed theoretical model both for the assertion of its general validity as well as for pointing out its possible improvements. To start with, all our results follow from the dielectric function expression of Eq. (1) which is obtained within the independent particle (i.e., random phase) approximation, ${ }^{46}$ and as such, it neglects the excitonic effects. In the case of intrinsic InN dielectric function, it has been shown by Furthmüller et al. that, apart from small shifts in the energy positions, these excitonic effects are responsible for the enhancement of some of the peaks in the spectra, predominantly close to the band edge, while the general shape remaining as unaffected. ${ }^{32}$ Furthermore, in the presence of high concentration of free carriers, as considered in this work, the excitonic effects get perfectly screened. The transition from bound excitonic state to free electron-hole plasma takes place at the Mott critical density. ${ }^{37}$ If we apply its estimate from Ref. 15 , we get about $5 \times 10^{16}$ and 5 $\times 10^{18} \mathrm{~cm}^{-3}$ for $\mathrm{InN}$ and $\mathrm{GaN}$, respectively. Above these values, excitons will dissociate which is easily met for the density range of this study.

On the BGR, there are other theoretical estimations, such as the plasmon-pole ${ }^{47}$ and the Vashishta-Kalia ${ }^{48}$ models. However, we observed that when contrasted with the existing experimental data on GaN (Ref. 49) and $\mathrm{InN}^{50}$ the employed Berggren-Sernelius expression ${ }^{34}$ in this and recent studies ${ }^{7,8}$ outperforms these other models in the relevant high density range of this study. From a formal point of view, there exist more rigorous many-body approaches which incorporate excitons, BGR and band-filling effects in the same level, however, their formidable computational cost leads to a trade off with a simplistic effective mass band structure..$^{51}$

Furthermore, we should mention that the employed Berggren-Sernelius expression assumes a cubic crystal with parabolic bands. ${ }^{34}$ These simplifications have been avoided by Persson and co-workers who have incorporated the electron-phonon contribution as well. ${ }^{40}$ Their framework is particularly better suited for handling the BGR under photoexcitation where both electron- and hole-gas many-body contributions can be individually included. However, as we stated previously, in the photoexcited case we still use the Berggren-Sernelius expression but with the reduced electronhole effective mass. Essentially, the same discussion also applies for the plasma contribution in Eq. (5).

Compared to the available $a b$ initio results for InN, 2,24,25,32 our band structure is in good overall agreement. Nevertheless, there remain further discrepancies of most of these theoretical results from the experimental data. First of all, due to the fact that our computational band gap of 0.85 $\mathrm{eV}$ for $\mathrm{InN}$ is somewhat higher than the experimentally es- tablished value, ${ }^{1}$ we expect especially our $1.55 \mu \mathrm{m}$ wavelength data to be affected. However, this change should be more or less similar to the deviation between 1.55 and $1.3 \mu \mathrm{m}$ cases in Fig. 13. In the case of GaN, our observation of a substantial deviation from the measured absorption in the vicinity of band gap (see, Fig. 3) calls for improvement on pseudopotential form factors for $\mathrm{GaN}$ which were originally optimized for high-field transport applications. ${ }^{22}$ Furthermore, we do not include the band tail (Urbach's tail) absorption from the impurity states forming bands below the band edge. ${ }^{52,53}$ Just like the excitons, the effect of localized states become marginal for high free-carrier densities, like beyond $10^{18} \mathrm{~cm}^{-3}$. Moreover, the characteristic Urbach energy parameter in high-quality $\mathrm{InN}$ samples turn out to be as low as 7.5 to $15 \mathrm{meV}$ which indicates that these features are anchored to just below the band gap. ${ }^{54}$

Being intended as a bulk study, this work neglects any surface effects, the most important of which is the surface charge accumulation causing built-in electric field and band bending. ${ }^{55,56}$ In this regard, it has been mentioned by Kudrawiec et al. that for $n$-type InN layer since the region of strong band bending is the surface electron accumulation layer, it will not affect absorptionlike techniques such as photoreflectance spectroscopy due to band filling of the conduction band. ${ }^{57}$ Another effect discarded in this study is the thermal smearing of the degenerate carrier statistics. As a possible follow up of this work it can be readily included, nevertheless we do not expect any marked deviations. Even though the quasi-Fermi level shift in the valence band is of the same order as the thermal energy (cf., inset of Fig. 5), as shown above, the main effect is driven by the Fermi level shift in the conduction band, which by far outweighs any thermal energy well up to room temperature for the carrier densities important for this work. Stemming from these idealizations, our main results in Fig. 13 should be taken as upper bounds. The list of these simplifications also suggests possible improvements of this work.

To harness the carrier-induced refractive index change such as in phase modulators, the associated losses need to be minimized. In this respect, the narrow $0.64 \mathrm{eV}$ band gap of InN becomes undesirable for the 1.55 and $1.3 \mu \mathrm{m}$ applica-

TABLE I. Fitted values for the constants in the pseudopotential form factors $V_{s}$ and $V_{a}$; see Eqs. (1) and (2). Other parameters used in the EPM are also listed, where $a$ and $c$ are the lattice constants in the hexagonal plane and along the $c$ axis, respectively, $u$ is the wurtzite internal structural parameter, and $E_{\max }$ denotes the radius of the energy sphere used for the reciprocal lattice vectors.

\begin{tabular}{ll}
\hline \hline$s_{1}=0.261905$ & $a_{1}=-0.411320$ \\
$s_{2}=0.232300$ & $a_{2}=0.176416$ \\
$s_{3}=-2.726784$ & $a_{3}=1.441420$ \\
$s_{4}=0.973335$ & $a_{4}=0.915637$ \\
$s_{5}=2.414422$ & $a_{5}=2.595997$ \\
$a=3.544 \AA$ & \\
$c=5.716472 \AA$ & \\
$u=0.379$ & \\
$E_{\max }=12.5$ Ry & \\
\hline
\end{tabular}


TABLE II. Fitted nonlocal EPM parameters for InN. $A$ values are in Rydbergs and $R$ values are in Angstroms.

\begin{tabular}{ll}
\hline \hline$A_{p}^{\mathrm{In}}=5.55440$, & $R_{p}^{\mathrm{In}}=0.195168$ \\
$A_{d}^{\mathrm{In}}=4.51525$, & $R_{d}^{\mathrm{In}}=0.132069$ \\
$A_{p}^{N}=3.64897$, & $R_{p}^{N}=0.089251$ \\
\hline
\end{tabular}

tions as they enable supra band gap excitations where additional losses set in, especially for the photoexcited case. Our comparative study here suggests that the $\operatorname{In}_{x} \mathrm{Ga}_{1-x} \mathrm{~N}$ can enjoy both the high carrier-induced refractive index sensitivity while at the same time operating in the transparency region of the alloy. The poor sensitivity of GaN to carrier-induced refractive index change, as demonstrated by this work points toward the choice of indium-rich alloys.

\section{CONCLUSIONS}

We have shown that based on a full band theoretical analysis, under $n$-type doping, $\mathrm{InN}$ and $\mathrm{GaN}$ should display different dielectric characteristics. The absorption edge shift is masked in $\mathrm{GaN}$, whereas it is highly pronounced in $\mathrm{InN}$. For energies below $1 \mathrm{eV}$, the corresponding shifts in the real parts of the dielectric function for $\mathrm{InN}$ and $\mathrm{GaN}$ are in opposite directions. The free-carrier plasma contribution to refractive index becomes dominant above $10^{20} \mathrm{~cm}^{-3}$ for the case of InN. At a wavelength of $1.55 \mu \mathrm{m}$, we predict more than $4 \%$ of change in the refractive index for InN for a doping of $10^{19} \mathrm{~cm}^{-3}$. Under optical pumping which fills the conduction and valence band edges with electrons and holes of equal density, the refractive index is not further affected by the presence of the holes. However, its major consequence is the increased intravalence band absorption as a result of $\Gamma_{5}^{v}$ $\rightarrow \Gamma_{6}^{v}$ transition. Beyond a hole density of $10^{19} \mathrm{~cm}^{-3}$, this affects 1.3 to $1.55 \mu \mathrm{m}$ wavelengths. We believe that this wide tunability of the index of refraction in $\mathrm{InN}$ by the conduction band electrons is technologically important for applications such as optical phase modulators. In the presence of holes, the valence band absorption needs to be taken into account, such as in the design of InN-based lasers for fiberoptics applications. Our findings suggest that the alloy com- position of $\operatorname{In}_{x} \mathrm{Ga}_{1-x} \mathrm{~N}$ can be optimized in the indium-rich region so as to benefit from high carrier-induced refractive index change while operating in the transparency region to minimize the losses.

\section{ACKNOWLEDGMENTS}

The authors would like to thank the British Council for supporting the partnership of the Essex and Bilkent Universities. CB and CMT would like to thank the EU FP7 Project UNAM-Regpot Grant No. 203953 for partial support.

\section{APPENDIX: EMPIRICAL PSEUDOPOTENTIAL PARAMETERS FOR WURTZITE InN}

In the light of recent experimental and first-principles studies on wurtzite InN, we have expressed the local empirical pseudopotential method (EPM) form factors using the following functional form,

$$
\begin{aligned}
& V_{s}(q)=\left(s_{1} q^{3}+s_{2} q^{2}+s_{3}\right) \exp \left(-s_{4} q^{s_{5}}\right), \\
& V_{a}(q)=\left(a_{1} q^{2}+a_{2} q+a_{3}\right) \exp \left(-a_{4} q^{a_{5}}\right),
\end{aligned}
$$

where $V_{s}$ and $V_{a}$ are the symmetric and antisymmetric form factors in Rydbergs, $q$ is the wave number in units of $2 \pi / a$ with $a$ being the lattice constant in the hexagonal plane, and $s_{i}, a_{i}(i=1, \ldots, 5)$ are the fitting parameters which are listed in Table I.

The nonlocal part of the pseudopotential is based on the classical work of Chelikowsky and Cohen, ${ }^{58}$ with the planewave matrix elements being given by

$$
\begin{aligned}
V_{N L}\left(\vec{K}, \vec{K}^{\prime}\right)= & \frac{4 \pi}{\Omega_{a}} \sum_{l, i} A_{l}^{i}(E)(2 l+1) P_{l}\left[\cos \left(\theta_{K K^{\prime}}\right)\right] S^{i}(\vec{K} \\
& \left.-\vec{K}^{\prime}\right) F_{l}^{i}\left(K, K^{\prime}\right),
\end{aligned}
$$

where $S^{i}(q)$ is the structure factor ${ }^{58}$ for the atomic species $i$, and $P_{l}(x)$ is the Legendre polynomial corresponding to angular-momentum channel $l, \Omega_{a}$ is the atomic volume (i.e., primitive cell volume divided by number of atoms), $\vec{K}=\vec{k}$ $+\vec{G}, \vec{K}^{\prime}=\vec{k}+\vec{G}^{\prime}$, and

$$
F_{l}\left(K, K^{\prime}\right)= \begin{cases}\frac{R^{3}}{2}\left\{\left[j_{l}(K R)\right]^{2}-j_{l-1}(K R) j_{l+1}(K R)\right\}, & K=K^{\prime}, \\ \frac{R^{2}}{K^{2}-K^{\prime 2}}\left[K j_{l+1}(K R) j_{l}\left(K^{\prime} R\right)-K^{\prime} j_{l+1}\left(K^{\prime} R\right) j_{l}(K R)\right], & K \neq K^{\prime}\end{cases}
$$

here, $j_{l}(x)$ is the spherical Bessel function, $R$ is (angular-momentum-dependent) well width. For InN, we treat the angularmomentum channels $p$ and $d$ for In, and $p$ for $\mathrm{N}$ as nonlocal. Table II lists InN nonlocal EPM parameters. 
*bulutay@fen.bilkent.edu.tr

${ }^{1}$ J. Wu, J. Appl. Phys. 106, 011101 (2009).

${ }^{2}$ P. Rinke, M. Winkelnkemper, A. Qteish, D. Bimberg, J. Neugebauer, and M. Scheffler, Phys. Rev. B 77, 075202 (2008).

${ }^{3}$ E. Burstein, Phys. Rev. 93, 632 (1954).

${ }^{4}$ T. S. Moss, Proc. Phys. Soc. London, Sect. B 67, 775 (1954).

${ }^{5}$ J. Wu, W. Walukiewicz, K. M. Yu, J. W. Ager III, E. E. Haller, H. Lu, W. J. Schaff, Y. Saito, and Y. Nanishi, Appl. Phys. Lett. 80, 3967 (2002).

${ }^{6}$ V. Yu. Davydov, A. A. Klochikhin, R. P. Seisyan, V. V. Emtsev, S. V. Ivanov, F. Bechstedt, J. Furthmüller, H. Harima, A. V. Mudryi, J. Aderhold, O. Semchinova, and J. Graul, Phys. Status Solidi B 229, r1 (2002).

${ }^{7}$ J. Wu, W. Walukiewicz, W. Shan, K. M. Yu, J. W. Ager, E. E. Haller, H. Lu, and W. J. Schaff, Phys. Rev. B 66, 201403(R) (2002).

${ }^{8}$ J. Wu, W. Walukiewicz, S. X. Li, R. Armitage, J. C. Ho, E. R. Weber, E. E. Haller, H. Lu, W. J. Schaff, A. Barcz, and R. Jakiela, Appl. Phys. Lett. 84, 2805 (2004).

${ }^{9}$ J.-F. Vinchant, J. A. Cavaillès, M. Erman, P. Jarry, and M. Renaud, J. Lightwave Technol. 10, 63 (1992).

${ }^{10}$ D. G. Deppe, N. D. Gerrard, C. J. Pinzone, R. D. Dupuis, and E. F. Schubert, Appl. Phys. Lett. 56, 315 (1990).

${ }^{11}$ J.-P. Weber, IEEE J. Quantum Electron. 30, 1801 (1994).

${ }^{12}$ P. A. Kirkby, A. R. Goodwin, G. H. B. Thompson, and P. R. Selway, IEEE J. Quantum Electron. 13, 705 (1977).

${ }^{13}$ A. Olsson and C. L. Tang, Appl. Phys. Lett. 39, 24 (1981).

${ }^{14}$ N. K. Dutta, N. A. Olsson, and W. T. Tsang, Appl. Phys. Lett. 45, 836 (1984).

${ }^{15}$ B. R. Bennett, R. A. Soref, and J. A. Del Alamo, IEEE J. Quantum Electron. 26, 113 (1990).

${ }^{16}$ L. Chusseau, P. Martin, C. Brasseur, C. Alibert, P. Hervé, P. Arguel, and F. Lozes-Dupuy, Appl. Phys. Lett. 69, 3054 (1996).

${ }^{17}$ P. P. Paskov, J. Appl. Phys. 81, 1890 (1997).

${ }^{18}$ W. W. Chow, H. Amano, and I. Akasaki, Appl. Phys. Lett. 76, 1647 (2000).

${ }^{19}$ U. T. Schwarz, E. Sturm, W. Wegscheider, V. Kümmler, A. Lell, and V. Härle, Appl. Phys. Lett. 83, 4095 (2003).

${ }^{20}$ M. Röwe, P. Michler, J. Gutowski, V. Kümmler, A. Lell, and V. Härle, Phys. Status Solidi A 200, 135 (2003).

${ }^{21}$ C. Bulutay and N. A. Zakhleniuk, Phys. Status Solidi C 5, 511 (2008).

${ }^{22}$ C. Bulutay, B. K. Ridley, and N. A. Zakhleniuk, Phys. Rev. B 62, 15754 (2000)

${ }^{23}$ C. Bulutay and B. K. Ridley, Superlattices Microstruct. 36, 465 (2004).

${ }^{24}$ P. Carrier and S.-H. Wei, J. Appl. Phys. 97, 033707 (2005).

${ }^{25}$ D. Bagayoko and L. Franklin, J. Appl. Phys. 97, 123708 (2005).

${ }^{26}$ J. L. P. Hughes and J. E. Sipe, Phys. Rev. B 53, 10751 (1996).

${ }^{27}$ R. M. Martin, Electronic Structure (Cambridge University Press, Cambridge, England, 2004).

${ }^{28}$ G. Lehmann and M. Taut, Phys. Status Solidi B 54, 469 (1972).

${ }^{29}$ D. J. Moss, J. E. Sipe, and H. M. van Driel, Phys. Rev. B 36, 1153 (1987).

${ }^{30}$ R. Goldhahn, A. T. Winzer, V. Cimalla, O. Ambacher, C. Cobet, W. Richter, N. Esser, J. Furthmüller, F. Bechstedt, H. Lu, and W. J. Schaff, Superlattices Microstruct. 36, 591 (2004).

${ }^{31}$ L. X. Benedict, T. Wethkamp, K. Wilmers, C. Cobet, N. Esser, E. L. Shirley, W. Richter, and M. Cardona, Solid State Commun. 112, 129 (1999).
${ }^{32}$ J. Furthmüller, P. H. Hahn, F. Fuchs, and F. Bechstedt, Phys. Rev. B 72, 205106 (2005).

${ }^{33}$ H. Jin, G. L. Zhao, and D. Bagayoko, J. Appl. Phys. 101, 033123 (2007).

${ }^{34}$ K.-F. Berggren and B. E. Sernelius, Phys. Rev. B 24, 1971 (1981).

${ }^{35}$ T. Azuhata, T. Sota, K. Suzuki, and S. Nakamura, J. Phys.: Condens. Matter 7, L129 (1995).

${ }^{36}$ A. Zubrilov, in Properties of Advanced Semiconductor Materials $G a N, A l N, \operatorname{InN}, B N, S i C, S i G e$, edited by M. E. Levinshtein, S. L. Rumyantsev, and M. S. Shur (Wiley, New York, 2001), pp. 49-66.

${ }^{37}$ C. F. Klingshirn, Semiconductor Optics, 3rd ed. (Springer, Berlin, 2007).

${ }^{38}$ M. Yoshikawa, M. Kunzer, J. Wagner, H. Obloh, P. Schlotter, R. Schmidt, N. Herres, and U. Kaufmann, J. Appl. Phys. 86, 4400 (1999).

${ }^{39}$ H. D. P. Schenk, S. I. Borenstain, A. Berezin, A. Schön, E. Cheifetz, S. Khatsevich, and D. H. Rich, J. Appl. Phys. 103, 103502 (2008).

${ }^{40}$ C. Persson, B. E. Sernelius, A. Ferreira da Silva, C. Moyseś Araújo, R. Ahuja, and B. Johansson, J. Appl. Phys. 92, 3207 (2002).

${ }^{41}$ V. A. Tyagai, A. M. Evstigneev, A. N. Krasiko, A. F. Andreeva, and V. Ya. Malakhov, Sov. Phys. Semicond. 11, 1257 (1977).

${ }^{42}$ T. Inushima, T. Yaguchi, A. Nagase, A. Iso, and T. Shiraishi, Inst. Phys. Conf. Ser. 142, 971 (1996); T. Inushima, T. Shiraishi, and V. Yu. Davydov, Solid State Commun. 110, 491 (1999).

${ }^{43}$ D. B. Haddad, J. S. Thakur, V. M. Naik, G. W. Auner, R. Naik, and L. E. Wenger, Mater. Res. Soc. Symp. Proc. 743, L11.22 (2002).

${ }^{44}$ There exists a somewhat different use of the "photodoping" terminology to describe the dissolution of metals, such as Ag or $\mathrm{Cu}$, into chalcogenide glasses under an illumination. However, this is more appropriately termed as photodissolution (Ref. 59).

${ }^{45}$ F. Chen, A. N. Cartwright, H. Lu, and W. J. Schaff, Appl. Phys. Lett. 83, 4984 (2003).

${ }^{46}$ H. Ehrenreich and M. H. Cohen, Phys. Rev. 115, 786 (1959).

${ }^{47}$ H. Haug and S. W. Koch, Phys. Rev. A 39, 1887 (1989).

${ }^{48}$ P. Vashishta and R. K. Kalia, Phys. Rev. B 25, 6492 (1982).

${ }^{49}$ T. Nagai, T. J. Inagaki, and Y. Kanemitsu, Appl. Phys. Lett. 84, 1284 (2004).

${ }^{50}$ P. Schley, R. Goldhahn, A. T. Winzer, G. Gobsch, V. Cimalla, O. Ambacher, H. Lu, W. J. Schaff, M. Kurouchi, Y. Nanishi, M. Rakel, C. Cobet, and N. Esser, Phys. Rev. B 75, 205204 (2007).

${ }^{51}$ J. P. Löwenau, S. Schmitt-Rink, and H. Haug, Phys. Rev. Lett. 49, 1511 (1982); J. P. Löwenau, F. M. Reich, and E. Gornik, Phys. Rev. B 51, 4159 (1995).

${ }^{52}$ W. Z. Shen, L. F. Jiang, H. F. Yang, F. Y. Meng, H. Ogawa, and Q. X. Guo, Appl. Phys. Lett. 80, 2063 (2002).

${ }^{53}$ B. Arnaudov, T. Paskova, P. P. Paskov, B. Magnusson, E. Valcheva, B. Monemar, H. Lu, W. J. Schaff, H. Amano, and I. Akasaki, Phys. Rev. B 69, 115216 (2004).

${ }^{54}$ A. Klochikhin, V. Davydov, V. Emtsev, A. Sakharov, V. Kapitonov, B. Andreev, H. Lu, and W. J. Schaff, Phys. Status Solidi A 203, 50 (2006).

${ }^{55}$ H. Lu, W. J. Schaff, L. F. Eastman, and C. E. Stutz, Appl. Phys. Lett. 82, 1736 (2003). 
${ }^{56}$ I. Mahboob, T. D. Veal, C. F. McConville, H. Lu, and W. J. Schaff, Phys. Rev. Lett. 92, 036804 (2004).

${ }^{57}$ R. Kudrawiec, T. Suski, J. Serafinćzuk, J. Misiewicz, D. Muto, and Y. Nanishi, Appl. Phys. Lett. 93, 131917 (2008).
${ }^{58}$ J. R. Chelikowsky and M. L. Cohen, Phys. Rev. B 14, 556 (1976).

${ }^{59}$ A. V. Kolobov and S. R. Elliott, Adv. Phys. 40, 625 (1991). 